\title{
Design of a film surface roughness-minimizing molecular beam epitaxy process by reduced-order modeling of epitaxial growth
}

\author{
Martha A. Gallivan a) and Harry A. Atwater \\ Thomas J. Watson Laboratory of Applied Physics, California Institute of Technology, Pasadena, \\ California 91125
}

(Received 30 June 2003; accepted 20 October 2003)

\begin{abstract}
Molecular beam epitaxy of germanium was used along with kinetic Monte Carlo simulations to study time-varying processing parameters and their effect on surface morphology. Epitaxial Ge films were deposited on highly oriented $\mathrm{Ge}(001)$ substrates, with reflection high-energy electron diffraction as a real-time sensor. The Monte Carlo simulations were used to model the growth process, and physical parameters were determined during growth under time-varying flux. A reduced version of the simulations was generated, enabling the application on an optimization algorithm. Temperature profiles were then computed that minimize surface roughness subject to various experimental constraints. The final roughness after two layers of growth was reduced to 0.32 , compared to 0.36 at the maximum growth temperature. The study presented here is an initial demonstration of a general approach that could also be used to optimize properties in other materials and deposition processes. (C) 2004 American Institute of Physics. [DOI: 10.1063/1.1632554]
\end{abstract}

\section{INTRODUCTION}

The wide range of existing thin film deposition techniques produce a vast array of film microstructure, composition, and purity. ${ }^{1}$ The final film properties are dependent on the source material, and, when the evolution is dominated by kinetic processes, ${ }^{2,3}$ are also dependent on the processing history. Process parameters, like temperature and precursor flux, can either be held constant or may be varied in time $e^{4,5}$ to produce the desired composition and surface morphology. The effect of periodic conditions on surface morphology has been studied in molecular beam epitaxy (MBE) of Group IV semiconductors, for both silicon and germanium homoepitaxy. ${ }^{6,7}$ Even in these simple single-species systems, the effect of pulsed flux on surface roughness is not straightforward. Depending on the growth strategy, pulsed flux may either be roughening ${ }^{7}$ or smoothing ${ }^{6}$ relative to growth at the mean flux.

To systematically investigate the relationship between growth strategies and surface morphology, parameter studies have been performed using kinetic Monte Carlo (KMC) simulations. ${ }^{7-9}$ Microscopic surface transitions are defined (e.g., adsorption, desorption, and surface diffusion), whose rates depend on physical parameters like vibrational frequencies, activation energies, and sticking coefficients. The Monte Carlo simulations are computationally intensive, so it is only practical to consider a small number of settings, for both the physical parameters and the process parameters. To study pulsed flux, Combe and Jensen ${ }^{8}$ varied pulse frequency and amplitude with and without adatom detachment from islands, while Taylor and Atwater ${ }^{7}$ compared constant and pulsed flux with and without energetic flux for a range of

\footnotetext{
a) Present address: School of Chemical and Biomolecular Engineering, Georgia Institute of Technology, Atlanta, GA 30332; electronic mail: martha.gallivan@chbe.gatech.edu
}

surface temperatures. Schinzer et $_{\text {al. }}{ }^{9}$ instead studied the effect of pulsed flux with and without desorption, in the presence of a step-edge barrier to surface diffusion.

Even when attention is restricted to periodic conditions, it is difficult to develop a comprehensive understanding of the relationship among all the physical parameters and all the processing parameters. We advocate a different approach here, in which a formal optimization method is used. Instead of simulating a number of growth strategies and comparing the resulting film properties, as in a parameter study, the desired film properties are first specified, after which the corresponding growth conditions are computed. A kinetic Monte Carlo simulation represents the growth of germanium on $\mathrm{Ge}(001)$, with physical parameters determined experimentally using reflection high-energy electron diffraction (RHEED). The computational demands of the KMC simulations are high, so a more compact representation is derived from KMC, using a method developed by one of the authors and described elsewhere. ${ }^{10}$ A gradient-based optimization algorithm is then applied to compute time-varying temperature profiles, and incorporates temperature and rate constraints associated with our MBE system.

The experimental work is described in Sec. II and the KMC simulations in Sec. III. Section IV contains the optimization, including the development of the compact reduced-order model. The optimized temperature profiles are discussed in Sec. V, along with the general applicability of our optimization approach.

\section{EXPERIMENT}

The experimental work was performed in a molecular beam epitaxy chamber for Group IV semiconductors. An electron-gun germanium source is located at the base of the chamber, positioned below the substrate block and manipulator. A computer-controlled pneumatic shutter is located be- 
tween the source and substrate block, enabling precise control over the deposition time. The substrate temperature is controlled with a tungsten-filament heater, which is calibrated periodically with a thermocouple-instrumented silicon wafer. A cryopump is used to maintain the typical base pressure of $1 \times 10^{-10}$ torr. The chamber also contains a reflection high-energy electron diffraction system, which is used to verify the initial $(2 \times 1)$ reconstruction of the germanium surface, and to monitor morphological evolution during deposition. An electron energy of $17 \mathrm{keV}$ is used for the RHEED measurements.

Germanium films were deposited on highly oriented $\mathrm{Ge}(001)$ wafers, specified by Eagle-Picher as $0.05^{\circ} \pm 0.02^{\circ}$. The wafers were prepared according to the following procedure. The wafers are cleaned by sonicating in acetone and methanol, followed by UV-ozone exposure and a dip in a 5\% HF solution. They are immediately loaded into the chamber, and annealed for $1-2 \mathrm{~h}$ at $250^{\circ} \mathrm{C}$. After this preparation, $(2 \times 1)$ surface reconstruction lines are visible in the RHEED pattern. The temperature is then raised to $550{ }^{\circ} \mathrm{C}$, and a $2000 \AA$ buffer layer of Ge is deposited at $1 \AA / \mathrm{s}$. The RHEED pattern is then dominated by the half circle of spots comprising the Ewald sphere, indicating that an atomically smooth surface has been attained.

Oscillations in the spectral beam spot during growth have been observed at a glancing angle of incidence of $0.5^{\circ} \cdot{ }^{11-13}$ Under certain conditions these oscillations may be a sensitive measure of film thickness, but they are not necessarily sensitive to surface morphology. ${ }^{12}$ In this paper, we present data taken at an angle of incidence of $5^{\circ}$. Oscillations are not observed in this configuration, but the intensity of the spectral spot is sensitive to the growth conditions.

For the experimental data presented here, growth conditions of $230-305^{\circ} \mathrm{C}$ and $0.05-0.8 \AA / \mathrm{s}$ were used, with a typical growth pressure of $5 \times 10^{-9}$ torr. Between each submonolayer deposition, the temperature was raised to $550^{\circ} \mathrm{C}$ for the growth of a $1000 \AA$ buffer layer at $1 \AA / \mathrm{s}$, to reattain the original RHEED pattern with the Ewald sphere. RHEED was also used as a real-time diagnostic during the lowtemperature growth. The intensity of the spectral beam spot was monitored using a photodiode, with an off-Bragg angle of incidence of $5^{\circ}$ and an azimuthal angle of $3^{\circ}$ from the (110) direction.

Figure 1 shows the normalized intensity of the spectral beam spot during growth and subsequent recovery. Typical intensity data are shown in Fig. 1(a), in which the intensity decays during growth and recovers after the source is shuttered. Figure 1(b) shows the results of a parameter study, in which $1 / 2$ monolayer (ML) is deposited, followed by $40 \mathrm{~s}$ of recovery during which islands may coarsen. The deposition rate and growth temperature are varied, and the normalized intensities are compared. The error bars reflect uncertainty in both the temperature and flux. The intensity decay for 0.4 $\AA / s$ is greater at all growth temperatures, relative to the intensities for $0.05 \AA / \mathrm{s}$. After $40 \mathrm{~s}$ of recovery at the higher growth rate, the intensity is similar to that immediately following deposition at the lower flux, across the entire range of temperatures. (a)

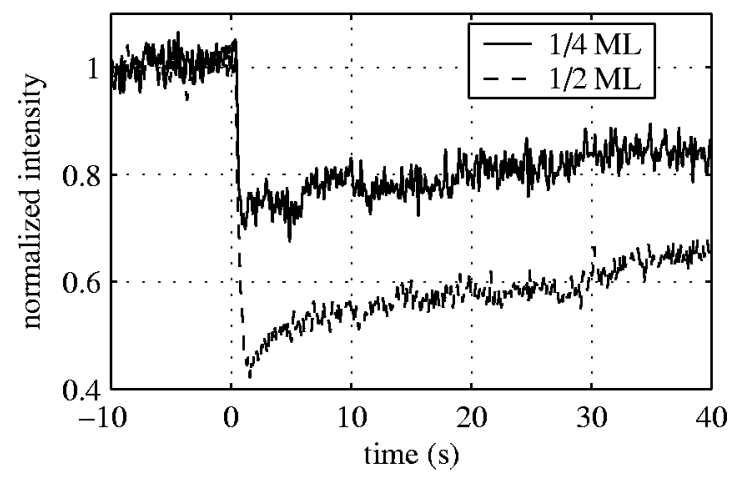

(b)

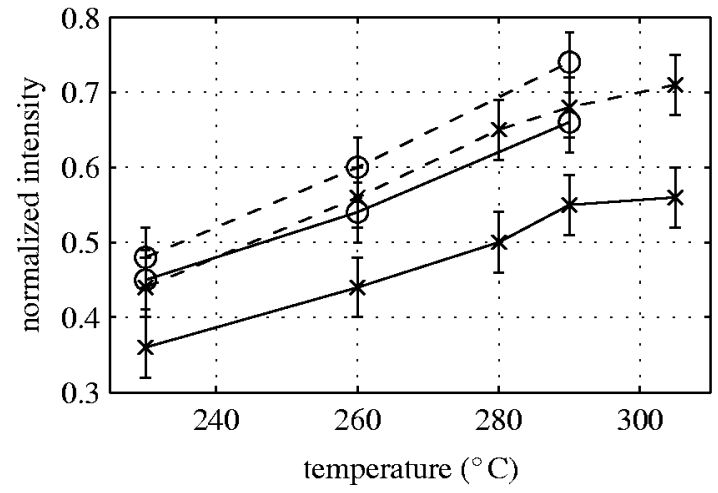

FIG. 1. Normalized intensity of RHEED spectral beam spot. (a) During deposition at $0.8 \AA / \mathrm{s}$ and $290^{\circ} \mathrm{C}$. (b) Immediately after the deposition of $1 / 2$ $\mathrm{ML}(\times)$ and after a further $40 \mathrm{~s}$ of annealing $(\mathrm{O})$, at $0.4 \AA / \mathrm{s}$ (solid line) and $0.05 \AA / s$ (dashed line).

\section{KINETIC MONTE CARLO SIMULATIONS}

We use kinetic Monte Carlo simulations ${ }^{14}$ as a model to describe the island nucleation, coarsening, and coalescence that characterize surface evolution on $\mathrm{Ge}(001)$. A cubic lattice, solid-on-solid assumption, and periodic boundary conditions are used, and a physical time is computed. ${ }^{15} \mathrm{An}$ inplane lattice parameter of $4.0 \AA$ and out-of-plane lattice parameter of $1.4 \AA$ reflect interatomic distances in germanium's diamond lattice. Adsorption and surface diffusion are simulated, with the adsorption rate equal to the incoming flux, and the diffusion rate for each atom dependent on the number of side bonds $i$, such that $k_{\mathrm{dif}, i}=\nu \exp \left[\left(E_{\mathrm{dif}, 0}\right.\right.$ $\left.+i \Delta E) /\left(k_{b} T\right)\right]$. This definition of the surface diffusion rate encompasses both adatom diffusion and detachment from islands. The step edge barrier for surface diffusion on $\mathrm{Ge}(001)$ has been reported negligible for the growth of very thin films, ${ }^{16}$ so we do not include it in our simulations.

\section{A. Parameter study}

Kinetic Monte Carlo simulations were performed for the experimental conditions corresponding to the data in Fig. 1(b). The vibrational frequency $\nu$ is taken as the Debye frequency of germanium, $7.8 \times 10^{12} \mathrm{~s}^{-1},{ }^{16}$ while the values of $E_{\text {dif }, 0}$ and $\Delta E$ are explored in simulation. The results are plotted in Fig. 2. Simulations are performed for $E_{\text {dif }, 0}$ $=0.65,0.70$, and $0.75 \mathrm{eV}$ and for $\Delta E=0.20,0.25$, and 0.30 $\mathrm{eV}$. The values of $E_{\mathrm{dif}, 0}$ are consistent with a previously reported activation energy for adatom diffusion $(0.65 \mathrm{eV}),{ }^{16}$ 

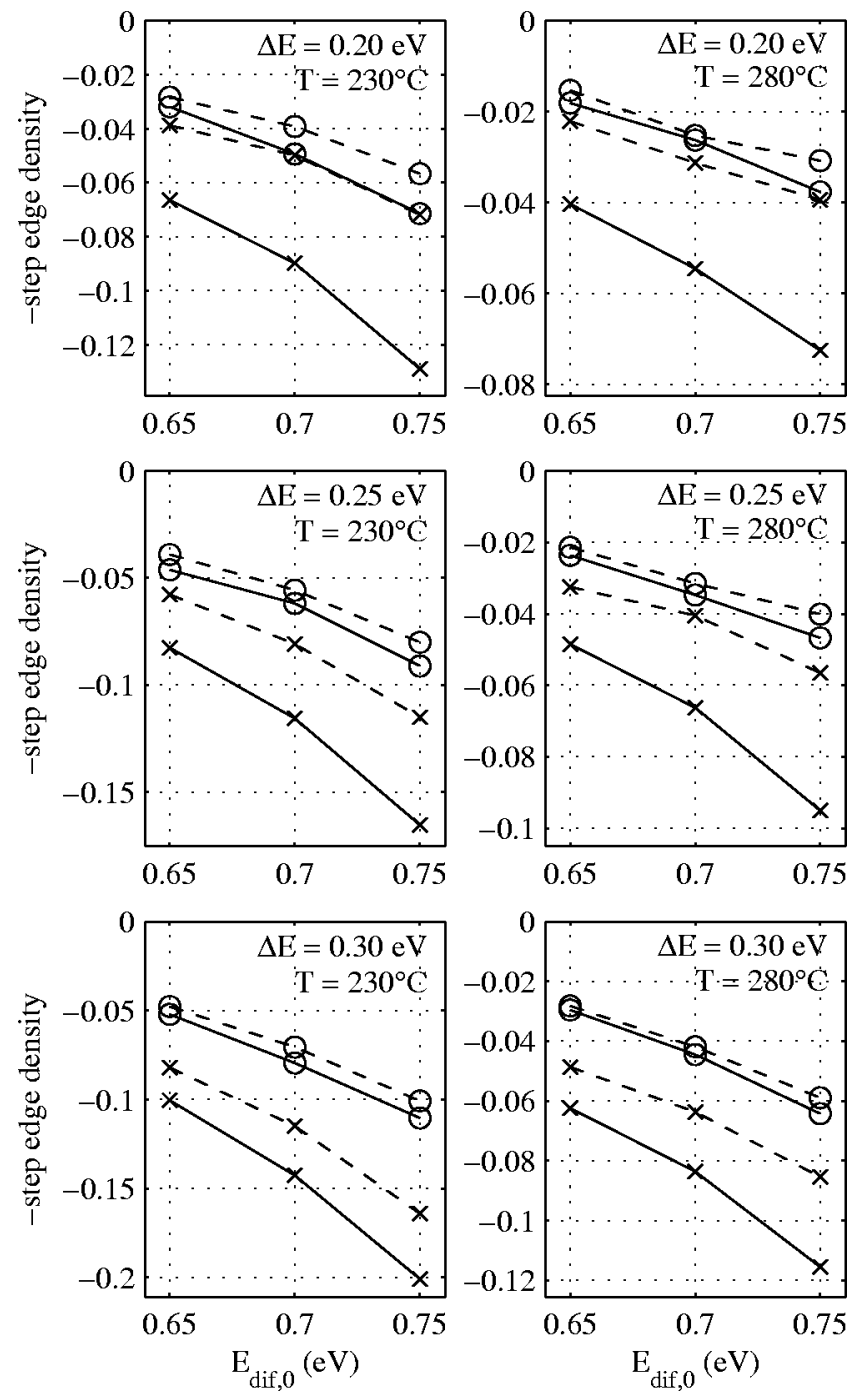

FIG. 2. KMC simulations of step edge density after the deposition of $1 / 2$ $\mathrm{ML}(\times)$ and after further $40 \mathrm{~s}$ of annealing $(\bigcirc)$, for $0.4 \AA / \mathrm{s}$ (solid line) and $0.05 \AA / s$ (dashed line). Two growth temperatures are simulated: $T$ $=230{ }^{\circ} \mathrm{C}$ (left) and $T=280{ }^{\circ} \mathrm{C}$ (right). The top pair of plots represents $\Delta E$ $=0.20 \mathrm{eV}$, the middle pair $\Delta E=0.25 \mathrm{eV}$, and the lower pair $\Delta E$ $=0.30 \mathrm{eV}$. In each plot, three values of $E_{\mathrm{dif}, 0}$ are simulated: $0.65,0.70$, and $0.75 \mathrm{eV}$.

while the three values of $\Delta E$ span the transition from negligible detachment of atoms from islands to substantial detachment, over the temperature range considered in this study.

\section{B. Comparison with experimental data}

The simulation data for step edge density is compared to the experimental data for spectral beam spot intensity by assuming an inverse monotonic relationship between the two quantities. The intensity of the spectral spot in the off-Bragg condition has been correlated with surface coverage ${ }^{17}$ and with step edge density ${ }^{18}$ using a kinematic approximation. In the former case, a quantitative relationship has been established for two-level growth ${ }^{19}$ and for stochastic growth. ${ }^{20}$ The validity of the latter step density model is the subject of active research-under certain circumstances, the intensity may actually increase with step density. ${ }^{21}$ In this paper we do not use any quantitative equation relating step density and

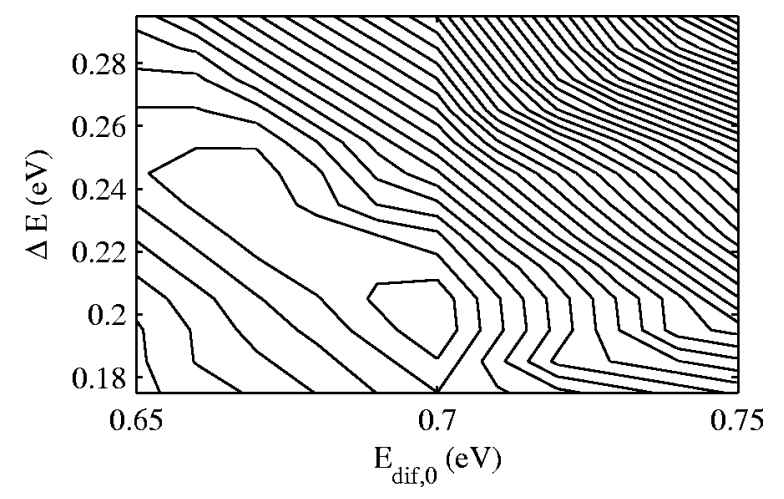

FIG. 3. Comparison of $\mathrm{Ge}(001)$ island density data from Van Nostrand et al. (Ref. 16) to our KMC simulations, at temperatures of 100,155 , and $230{ }^{\circ} \mathrm{C}$. Error is measured as the root mean square of the relative error in island density at each temperature. The contours, spaced at intervals of 0.05 , denote the error, beginning with 0.15 surrounding $E_{\mathrm{dif}, 0}=0.70 \mathrm{eV}$ and $\Delta E$ $=0.20 \mathrm{eV}$.

spectral spot intensity, but instead assume that for fixed temperature and fixed coverage, a surface with a higher step (and island) density has a lower spectral beam spot intensity. This interpretation is supported by the data in Fig. 2(b). For fixed temperature and coverage, a higher flux generates a greater decay in intensity, and, as predicted by rate equation models, ${ }^{22}$ also generates a higher step density.

This interpretation is used to compare the data of Fig. 2(b) with the kinetic Monte Carlo simulations. Thus, the simulations are consistent with the data if the simulated step density after growth at $0.4 \AA / s$ and 40 s of recovery is equal to that of the step density immediately following growth at $0.05 \AA / s$. The simulations of Fig. 2 satisfy this criterion with $\Delta E=0.20 \mathrm{eV}$, while those with $\Delta E=0.25 \mathrm{eV}$ or $0.30 \mathrm{eV}$ do not. At a lower value of $\Delta E=0.15 \mathrm{eV}$, compact islands were not observed over this temperature range.

The relative relationship between the simulation curves is insensitive to $E_{\mathrm{dif}, 0}$. To obtain an appropriate value for $E_{\mathrm{dif}, 0}$ we compared our KMC simulations to previously reported data for island densities on $\mathrm{Ge}(001) .{ }^{16}$ We simulated the deposition of $0.3 \mathrm{ML}$ at 100,155 , and $230^{\circ} \mathrm{C}$, the conditions under which the data were reported. The experimentally observed island densities were then compared to our simulated densities for a range of $E_{\mathrm{dif}, 0}$ and $\Delta E$. The percent error in the island density was computed at each temperature, and was used to compute the root-mean-square (rms) error for the three temperatures. This rms error is plotted in Fig. 3.

The best agreement between our simulations and the island density data of Van Nostrand et al. ${ }^{16}$ is obtained for $E_{\mathrm{dif}, 0}=0.70 \mathrm{eV}$ and $\Delta E=0.20 \mathrm{eV}$, with a rms error of 0.14 . Good agreement (error less than 0.20) is also obtained if a decrease in $E_{\mathrm{dif}, 0}$ is countered with an increase in $\Delta E$. In this case, the activation energy for dimer dissociation is unchanged, although the kink detachment energy is greater. However, raising the value of $\Delta E$ above $0.20 \mathrm{eV}$ is inconsistent with our RHEED data. We thus take $E_{\text {dif }, 0}=0.70 \mathrm{eV}$ and $\Delta E=0.20 \mathrm{eV}$ as the physical parameters in our Monte Carlo model.

We find it somewhat unexpected that the values of $E_{\mathrm{dif}, 0}=0.70 \mathrm{eV}$ and $\Delta E=0.20 \mathrm{eV}$ are consistent with the low 
temperature data between 100 and $230{ }^{\circ} \mathrm{C},{ }^{16}$ and our higher temperature data between 230 and $305^{\circ} \mathrm{C}$. The cubic lattice model would not necessarily capture morphological evolution over this wide temperature range, since it does not model the diamond lattice or specific microscopic transitions on this lattice. We conclude that the surface evolution may in fact be dominated by simple idealized mechanisms like adsorption, dimer dissociation, and kink detachment, which are well modeled by the cubic lattice simulations.

\section{Alternative growth strategies}

RHEED was used as the primary surface diagnostic in this study. Other in situ techniques, including scanning tunneling microscopy (STM) measurements, could provide additional information if available, but ex-situ characterization of the morphology is not practical since atomic-height features would be obscurred by surface oxidation. Our MBE experiments were performed at a sufficiently high temperature to ensure a straightforward interpretation of the specular spot beam intensity. For the same reason, the temperature was held constant during each deposition. In this temperature range, pulsing the flux in MBE using a shutter was not observed to alter the surface morphology in experiment or in simulation, even over several monolayers of growth. ${ }^{23} \mathrm{Ki}$ netic Monte Carlo simulations indicate that at these growth temperatures smooth surfaces are obtained even when there is no time separation between the flux pulses. Recent experimental studies also suggest that at our growth temperatures, epitaxial growth can be maintained indefinitely. ${ }^{24}$

We instead explore in simulation the effects of flux and temperature pulsing in growth at lower temperatures, with root-mean-square surface roughness used to characterize morphology. High instantaneous fluxes are explored, which are not attainable using our electron-beam source. Figure $4(\mathrm{a})$ is a study of pulsed flux, with a temperature of $150{ }^{\circ} \mathrm{C}$ and a mean growth rate of $1.4 \AA / \mathrm{s}(1 \mathrm{ML} / \mathrm{s})$. The flux is delivered in $5 \mathrm{~ms}$ pulses, reminiscent of pulsed laser deposition. ${ }^{7}$ At instantaneous fluxes accessible in MBE, no difference is observed relative to continuous growth, but when extreme pulses are used the surface roughens.

A different strategy is shown in Fig. 4(b). A continuous flux of $1.4 \AA / s$ is used, and a comparison is made between growth at a constant temperature of $150{ }^{\circ} \mathrm{C}$, and a strategy in which the temperature is lowered to $75^{\circ} \mathrm{C}$ during the first $20 \%$ of each layer to enhance island nucleation, after which it is raised to $150{ }^{\circ} \mathrm{C}$. Although a time-varying temperature could be implemented in our MBE chamber, it was not investigated here because it would also complicate the interpretation of RHEED, our only surface diagnostic. However, the simulations predict that the increased island density at the beginning of each layer delays the onset of secondary nucleation on top of islands, yielding a smoother interface at the end of each monolayer. This technique has been observed previously in experiment, ${ }^{6}$ where it is referred to as synchronization of nucleation. Based on the simulations in Fig. 4, we conclude that time-varying growth conditions may be either be roughening or smoothing, depending on the phase, frequency, and amplitude of the modulation. (a)
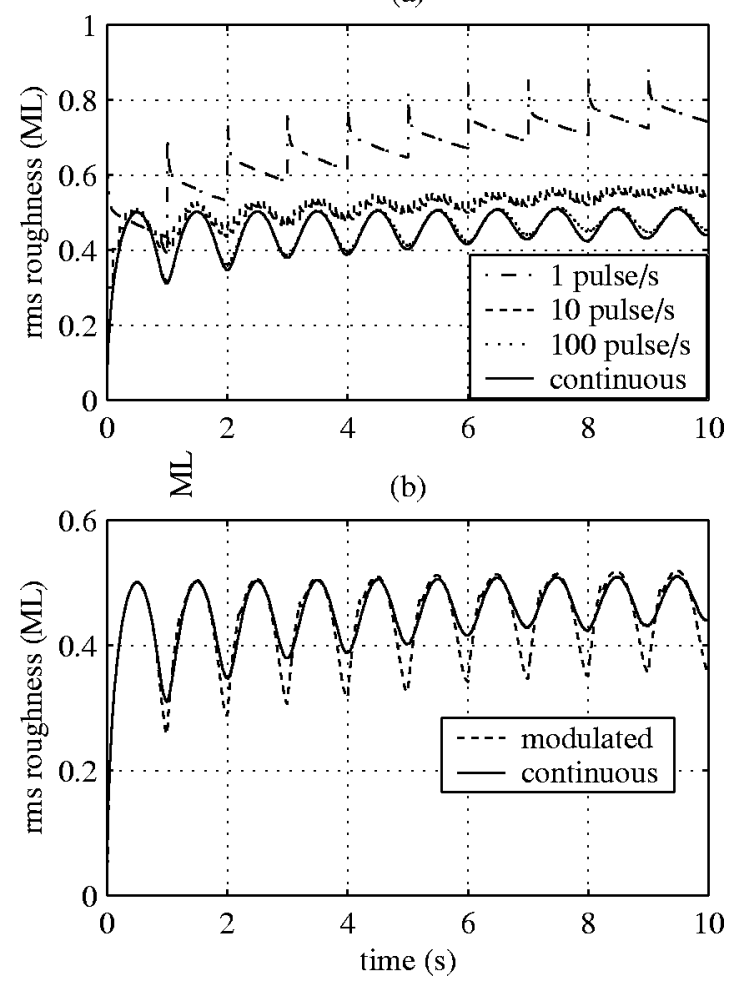

FIG. 4. Kinetic Monte Carlo simulations of periodic growth conditions: (a) continuous temperature of $150{ }^{\circ} \mathrm{C}$, with a mean flux of $1.4 \AA / \mathrm{s}$ and various duty cycles; (b) continuous flux of $1.4 \AA / \mathrm{s}$ and either a continuous temperature of $150{ }^{\circ} \mathrm{C}$ or a synchronized temperature that is lowered to $75^{\circ} \mathrm{C}$ during the first $20 \%$ of each monolayer.

\section{OPTIMIZATION}

Time-varying growth conditions may lead to altered surface properties, but it is not practical to try every possible combination in experiment, or even in KMC simulation. An alternate approach is to apply an optimization algorithm to compute the best time-varying conditions, using a model. The KMC simulations are computationally demanding and noisy, so it is also not practical to perform the many simulations needed for an optimization algorithm. We reduce these computational demands by computing a low-dimensional equation that captures the dynamics of the KMC simulation, using a method developed by one of the authors and documented elsewhere. ${ }^{10}$ This model is then used in a mathematical optimization algorithm.

\section{A. Model reduction}

The phrase model reduction refers to a concept and framework developed within the dynamics and control community. ${ }^{25,26}$ Its goal is to reduce the number of variables in a high-dimensional dynamic model, while still providing an accurate model for particular quantities of interest. For example, in the KMC simulations, one typically does not need to know the location of each atom, but does wish to describe the evolution of surface metrics like thickness, roughness, and island density. Systematic algorithms have been developed to generate reduced-order models from highdimensional simulations, ${ }^{25,26}$ and have been used to generate low-dimensional models for turbulent flow. ${ }^{27}$ 
(a)

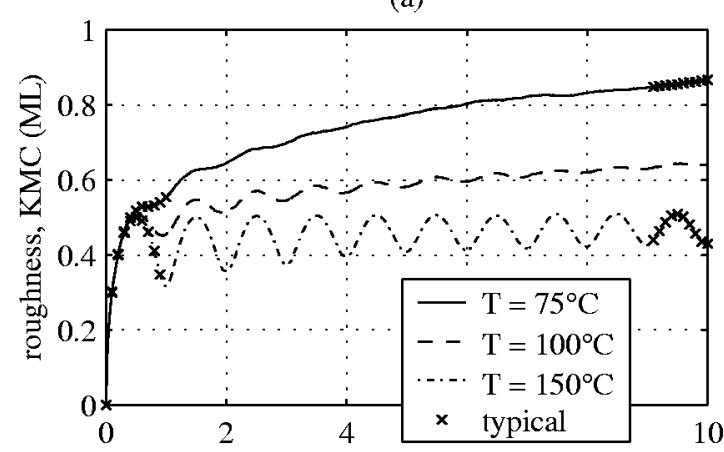

(b)

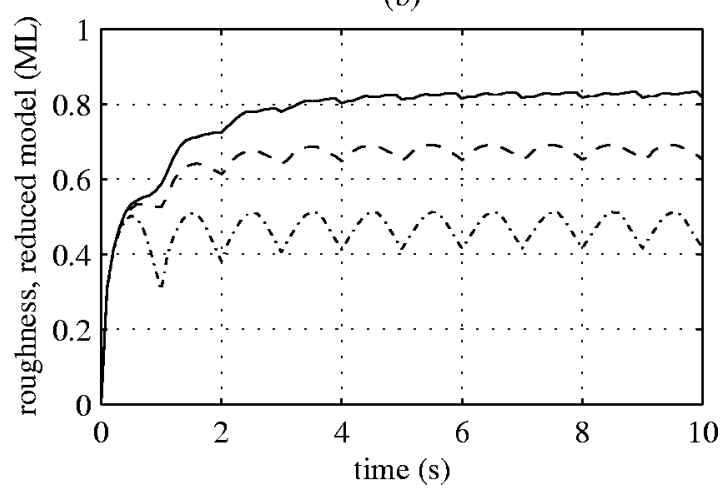

FIG. 5. Comparison of rms roughness for (a) KMC simulations and (b) reduced-order model. The $\times$ denote points in the simulation when microscopic configurations were extracted to represent typical surfaces.

The reduced-order model used here has the mathematical structure of an inhomogeneous hidden Markov chain, with KMC simulation data used to generate all its coefficients. ${ }^{10}$ A small number of surface configurations are first determined using physical arguments, with each configuration representing some typical combination of surface coverage, roughness, step density, and adatom density. Eighty configurations are selected for the model used in this study, to represent growth at $1.4 \AA$ As over 10 monolayers, for a temperature range of $75-150{ }^{\circ} \mathrm{C}$. The configurations are extracted from the KMC simulations plotted in Fig. 5(a). At each of the 40 points marked $\times$, the microscopic surface configuration is saved.

These 40 configurations are further modified to capture the high instantaneous adatoms densities observed during pulsed growth. Two new configurations are generated for each of the 40 original ones, by removing all adatoms, and by adding adatoms at random locations to produce an adatom density of $2.5 \times 10-3$ per site. (This "high" adatom density was observed in our pulsed growth simulations.) In the reduced-order model, each of the resulting 80 configurations represents the probability of being in some configuration with the same surface properties. Thus, the reduced model describes the probability evolution for 80 configuration groups.

The probabilities of being in each configuration group are computed by a Markov chain model, whose transition probabilities depend on the processing parameters. In this example, the only processing parameter to be varied is the temperature, and KMC simulations beginning in each of the

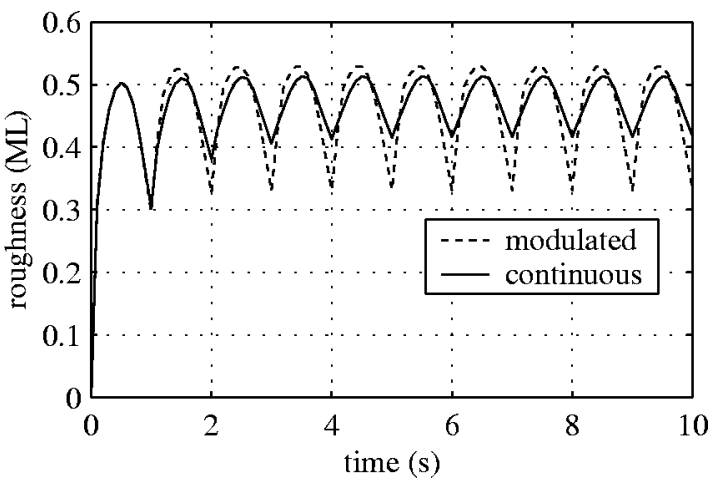

FIG. 6. Roughness evolution predicted by the reduced model under the same periodic growth conditions as for Fig. 4(b). The reduced model captures the smoothing effect of synchronized modulation, but overpredicts its magnitude.

80 configurations are performed to compute the temperaturedependent transition probabilities. The roughness evolution of the reduced model under constant temperature is plotted in Fig. 5(b). Perfect quantitative agreement between the KMC simulations and reduced model is not achieved, but the model does capture qualitative features like the roughening and loss of oscillation with decreasing temperature.

The reduced model was developed to make predictions for time-varying growth conditions. Figure 6 displays the reduced model simulations for the same growth strategy as pictured in Fig. 4(b). The reduced model captures the smoothing effect of modulated growth, relative to growth at the maximum temperature, captures the qualitative behavior, and has reasonable quantitative agreement.

\section{B. Optimization}

The reduced model is now used to compute temperature profiles that minimize the roughness after two layers of growth, using MATLAB'S FMINCON function. The temperature profile is divided into intervals of $0.1 \mathrm{~s}$, over which the temperature is held constant. Three optimized temperature profiles are shown in Fig. 7(a). In all three cases, the objective is to minimize the final roughness, while remaining in the range $75-150^{\circ} \mathrm{C}$, but different constraints are imposed on the rate of temperature change. Case 1 represents the thermal environment of our MBE chamber, in which the maximum heating rate is estimated at $50 \mathrm{~K} / \mathrm{s}$ in this temperature range, while the maximum cooling rate of $0.5 \mathrm{~K} / \mathrm{s}$ through radiation is much slower. Under these constraints it is not possible to lower the temperature significantly over $2 \mathrm{~s}$, so the best approach is to start with a low temperature to nucleate many islands, and then raise the temperature up to the maximum at the end. Note also that the temperature plateaus around the initiation of the second layer, presumably so that more islands will be nucleated. In case 2 , the maximum cooling rate is raised to the value of the maximum heating rate. Again, it is desirable to minimize the temperature at the initiation of each layer, and also to have the maximum temperature at the end. In case 3, no rate constraints are placed on the temperature, and the best strategy is to minimize the temperature at the beginning of a layer, and maximize it at the end. 
(a)

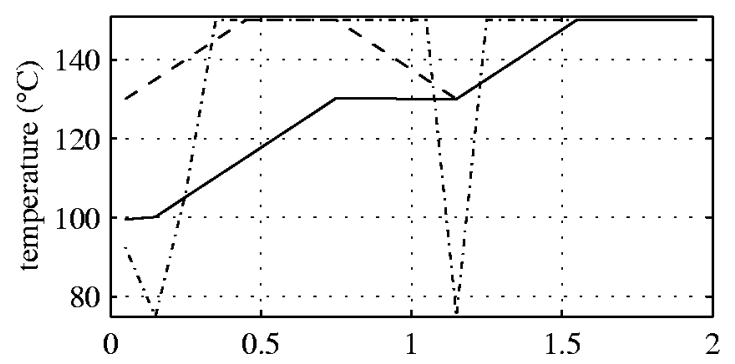

(b)

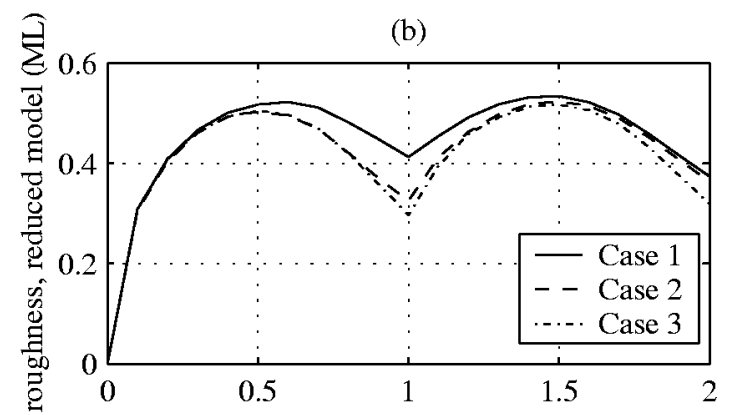

(c)

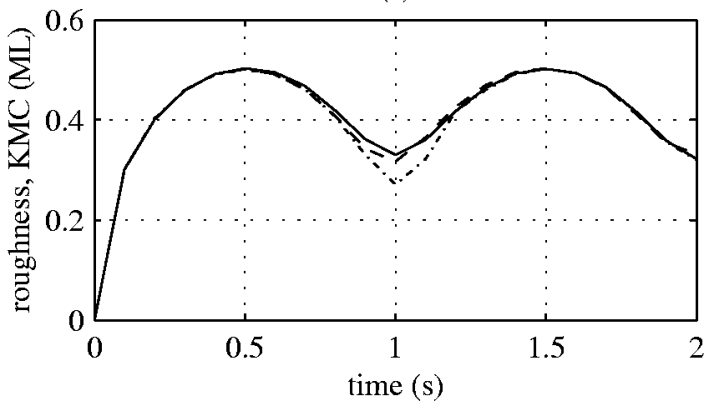

FIG. 7. (a) Optimal temperature profile computed using the reduced-order model, to minimize roughness after 2 monolayers of growth. These profiles are then simulated in (b) the reduced-order model and (c) the KMC simulations. Case 1: maximum heating rate of $50 \mathrm{~K} / \mathrm{s}$ and cooling rate of $0.5 \mathrm{~K} / \mathrm{s}$; case 2: maximum heating and cooling rates of $50 \mathrm{~K} / \mathrm{s}$; case 3: no constraints on temperature change.

When these temperature profiles are implemented in the reduced-order model, shown in Fig. 7(b), the minimum roughness is achieved with no rate constraints of temperature (case 3), while the constraints of cases 1 and 2 prevent achievement of the lowest roughness. Some error was introduced in reducing the KMC simulations, so when the optimized temperature profiles are implemented in KMC, the differences in final roughness between the three cases is negligible, as shown in Fig. 7(c). A quantitative comparison is made in Table I. In both models all three optimized temperature profiles generate a reduction in final roughness relative to the roughness predicted for growth at a continuous temperature of $150{ }^{\circ} \mathrm{C}$. Thus, the temperature profiles generated with the reduced models may not be truly optimal for the KMC simulations, but they do suggest temperature profiles that achieve the desired objective in KMC, to smooth the surface.

\section{DISCUSSION AND CONCLUSIONS}

In this work we have performed MBE experiments to study the nucleation and coarsening of islands in $\mathrm{Ge}(001)$
TABLE I. Comparison of final roughness for the three temperature profiles of Fig. 7(a), and for a constant profile of $150{ }^{\circ} \mathrm{C}$. The rms roughness after two monolayers of growth is reported using the reduced-order model and the original kinetic Monte Carlo simulations, also shown in Figs. 7(b) and 7(c).

\begin{tabular}{ccc}
\hline \hline & $\begin{array}{c}\text { Reduced-order } \\
\text { model }\end{array}$ & $\begin{array}{c}\text { KMC } \\
\text { simulations }\end{array}$ \\
\hline$T=150^{\circ} \mathrm{C}$ & 0.381 & 0.358 \\
Case 1 & 0.374 & 0.322 \\
Case 2 & 0.363 & 0.332 \\
Case 3 & 0.319 & 0.320 \\
\hline \hline
\end{tabular}

homoepitaxy. Real-time RHEED measurements were used as the diagnostic for surface morphology, and were compared to $\mathrm{KMC}$ simulations to determine the activation energies for surface diffusion on a cubic lattice. The KMC simulations were then used to make predictions of growth strategies that were not accessible in our MBE system (high instantaneous flux) or that would have obfuscated the interpretation of the RHEED signal (temperature variation). The simulations predict that, in low temperature growth, periodic growth conditions may produce either smoothing or roughening.

To study the effect of arbitrary time-varying growth conditions, a reduced-order model was generated using KMC simulation data. Temperature profiles that minimize surface roughness were then computed using an optimization algorithm, which incorporated the thermal dynamics of the MBE heater assembly. The reduced model enabled the application of an optimization algorithm, which was not practical using the full KMC simulation, and also enabled coupling between the microscopic dynamics of surface morphology and the macroscopic temperature dynamics of the MBE chamber. The roughness of the final surface was reduced to 0.32 , compared to 0.36 for growth at the maximum temperature. Benefits of this modeling and optimization approach include both the generation of a smoother interface, and a quantification of the best possible interface. Moreover, the approach presented here is general, and could be used to optimize material properties in systems other than germanium and by processes other than MBE.

Implementation of the optimized growth conditions in an MBE process remains a subject for future research. The simulation and optimization studies suggest that timevarying processing conditions can be beneficial, even subject to the current flux and temperature constraints. However, to test these in experiment, quantitative surface measurements are required, either with dynamical modeling of the RHEED signal, or with the use of additional surface diagnostics techniques, such as STM. The use of simulations and optimization also suggests chamber modifications that would make possible a wider range of growth conditions, such as fast switching between conditions. The integrated experimental, simulation, and modeling study presented here provides a unique approach to systematic process design that is made possible with a physics-based predictive model of surface evolution. 


\section{ACKNOWLEDGMENTS}

This work was supported by DARPA/NSF Grant No. DMS-9615858, Grant No. AFOSR-F49620-95-1-0419, Grant No. DAAD19-01-1-0517, NSF Grant No. ECS 0103543, and a NSF graduate fellowship. The authors thank Richard Murray for fruitful discussions, and Regina Ragan for support in the experimental work.

${ }^{1}$ Thin Film Technology Handbook, edited by A. Elshabini-Riad and F. D. Barlow III (McGraw-Hill, New York, 1998).

${ }^{2}$ Monte Carlo Methods in Statistical Physics, edited by K. Binder (Springer-Verlag, Berlin, 1979), pp. 261-300.

${ }^{3}$ Morphological Organization in Epitaxial Growth and Removal, edited by Z. Zhang and M. G. Lagally (World Scientific, Singapore, 1998).

${ }^{4}$ M. Svensson, U. Wahlstrom, and G. Holmbom, Surf. Coat. Technol. 105, 218 (1998)

${ }^{5}$ D. Yang, R. Jonnalagadda, B. R. Rogers, J. T. Hillman, R. F. Foster, and T. S. Cale, Thin Solid Films 332, 312 (1998).

${ }^{6}$ V. A. Markov, O. P. Pchelyakov, L. V. Sokolov, S. I. Stenin, and S. Stoyanov, Surf. Sci. 250, 229 (1991).

${ }^{7}$ M. E. Taylor and H. A. Atwater, Appl. Surf. Sci. 129, 159 (1998).

${ }^{8}$ N. Combe and P. Jensen, Phys. Rev. B 57, 15553 (1998).

${ }^{9}$ S. Schinzer, M. Sokolowski, M. Biehl, and W. Kinzel, Phys. Rev. B 60, 2893 (1999).

${ }^{10}$ M. A. Gallivan and R. M. Murray, Int. J. Robust Nonlin. Control 14, 113 (2004).
${ }^{11}$ J. Aarts, W. M. Gerits, and P. K. Larsen, Appl. Phys. Lett. 48, 931 (1986).

${ }^{12}$ J. Aarts, A. J. Hoeven, and P. K. Larsen, J. Vac. Sci. Technol. A 6, 607 (1988).

${ }^{13}$ C. L. Berrie and S. R. Leone, J. Cryst. Growth 216, 159 (2000).

${ }^{14}$ G. H. Gilmer and P. Bennema, J. Appl. Phys. 43, 1347 (1972).

${ }^{15}$ A. B. Bortz, M. H. Kalos, and J. L. Lebowitz, J. Comput. Phys. 17, 10 (1975)

${ }^{16}$ J. E. V. Nostrand, S. J. Chey, and D. G. Cahill, Phys. Rev. B 57, 12536 (1998).

${ }^{17}$ C. S. Lent and P. I. Cohen, Surf. Sci. 139, 121 (1984).

${ }^{18}$ T. Shitara, D. D. Vvedensky, M. R. Wilby, J. Zhang, J. H. Neave, and B. A. Joyce, Appl. Phys. Lett. 60, 1504 (1992).

${ }^{19}$ P. I. Cohen, P. R. Pukite, J. M. V. Hove, and C. S. Lent, J. Vac. Sci. Technol. A 4, 1251 (1986).

${ }^{20}$ E. Chason, J. Y. Tsao, K. M. Horn, and S. T. Picraux, J. Vac. Sci. Technol. A 7, 332 (1989).

${ }^{21}$ U. Korte and P. A. Maksym, Phys. Rev. Lett. 78, 2381 (1997).

${ }^{22}$ J. A. Venables, Philos. Mag. 27, 697 (1973).

${ }^{23}$ M. A. Gallivan, Ph.D. thesis, California Institute of Technology, Pasadena, CA 91125 (2002), http://etd.caltech.edu/etd/available/etd-10222002$115711 /$

${ }^{24}$ K. A. Bratland, Y. L. Foo, J. A. N. T. Soares, T. Spila, P. Desjardins, and J. E. Greene, Phys. Rev. B 67, 125322 (2003).

${ }^{25}$ B. C. Moore, IEEE Trans. Autom. Control 26, 1732 (1981).

${ }^{26}$ J. M. A. Scherpen, Syst. Control Lett. 21, 143 (1993).

${ }^{27}$ N. Aubry, P. Holmes, J. L. Lumley, and E. Stone, J. Fluid Mech. 192, 115 (1988). 